\title{
Comparing Bayesian estimates of genetic differentiation of molecular markers and quantitative traits: an application to Pinus sylvestris
}

\author{
P Waldmann ${ }^{1,3}$, MR García-Gil ${ }^{2,3}$ and MJ Sillanpää ${ }^{1}$ \\ ${ }^{1}$ Rolf Nevanlinna Institute, PO Box 68, FIN-000 14 University of Helsinki, Finland; ' ${ }^{2}$ epartment of Biology, University of Oulu, \\ PO Box 3000, FIN-900 14 University of Oulu, Finland; 'Department of Forest Genetics and Plant Physiology, SLU, SE-901 83 Umea, \\ Sweden
}

\begin{abstract}
Comparison of the level of differentiation at neutral molecular markers (estimated as $F_{\mathrm{ST}}$ or $G_{\mathrm{ST}}$ ) with the level of differentiation at quantitative traits (estimated as $Q_{\mathrm{ST}}$ ) has become a standard tool for inferring that there is differential selection between populations. We estimated $Q_{\mathrm{ST}}$ of timing of bud set from a latitudinal cline of Pinus sylvestris with a Bayesian hierarchical variance component method utilizing the information on the pre-estimated population structure from neutral molecular markers. Unfortunately, the betweenfamily variances differed substantially between populations that resulted in a bimodal posterior of $Q_{\mathrm{ST}}$ that could not be
\end{abstract}

compared in any sensible way with the unimodal posterior of the microsatellite $F_{\mathrm{ST}}$. In order to avoid publishing studies with flawed $Q_{\mathrm{ST}}$ estimates, we recommend that future studies should present heritability estimates for each trait and population. Moreover, to detect variance heterogeneity in frequentist methods (ANOVA and REML), it is of essential importance to check also that the residuals are normally distributed and do not follow any systematically deviating trends.

Heredity (2005) 94, 623-629. doi:10.1038/sj.hdy.6800672

Published online 27 April 2005

Keywords: genetic drift; selection; population structure; $Q_{\mathrm{ST}} ; F_{\mathrm{ST}}$; MCMC; Scots pine

\section{Introduction}

Many species occur in several or at least partly isolated subpopulations that are adapted to local environmental conditions. These populations are influenced by a number of forces that can alter their genetic structure. In general, genetic drift and diversifying selection will differentiate the gene frequencies, whereas gene flow, mutation and unifying selection will counteract these forces. Estimation of genetic differentiation between populations using molecular markers is an important topic in many areas of evolutionary science (Avise, 1993; Goldstein and Schlötterer, 1999). Most studies have used statistical measures derived from Wright's F-statistics (Wright (1951, 1965); see also Excoffier, 2001; Weir and Hill, 2002).

Given an island model, it has been shown that genetic differentiation between populations, expressed as Wright's $F_{\mathrm{ST}}$, should be the same regardless of whether it is estimated from neutral single-locus marker or from a neutral quantitative trait with an additive genetic basis (Lande, 1992; Lynch, 1994). Whitlock (1999) showed that this procedure can be applied also to other types of population structures. Hence, the amount of differentiation in neutral molecular markers $\left(F_{\mathrm{ST}}\right)$ can be used as an expectation for the level of differentiation of the neutral

Correspondence: P Waldmann, Department of Forest Genetics and Plant Physiology, SLU, SE-901 83 Umeå, Sweden.

E-mail:Patrik.Waldmann@genfys.slu.se

Received 26 September 2004; accepted 17 February 2005; published online 27 April 2005 additive genetic variance of a quantitative trait among populations (termed $Q_{\mathrm{ST}}$ by Spitze, 1993). If the value of $Q_{\mathrm{ST}}$ is larger than the corresponding value of $F_{\mathrm{ST}}$, one can conclude that there is some evidence against the neutrality hypothesis in favour of diversifying selection among the populations. Conversely, if the value of $Q_{\mathrm{ST}}$ is lower than $F_{\mathrm{ST}}$, one can say that unifying selection has been the prevalent force. This reasoning, of course, assumes that the mutation rates are the same at the loci involved. An increasing number of studies have compared the differentiation in molecular markers and quantitative traits, and it seems that quantitative traits generally are more differentiated than molecular markers (reviewed in Merilä and Crnokrak, 2001; Reed and Frankham, 2001; McKay and Latta, 2002). However, except for Palo et al (2003), $Q_{\mathrm{ST}}$ and $F_{\mathrm{ST}}$ estimates have so far not been compared in a statistically rigorous way.

Bayesian inferential methods have recently started to emerge for different areas in evolutionary biology (Beaumont and Rannala, 2004). They provide a number of advantages compared to classical frequentist approaches. One such advantage is a probabilistic measure of uncertainty in the form of credible intervals (CI), which can be obtained on parameter estimates and even on their functions. By contrast, maximum likelihood methods only provide standard errors around the parameters in the model (from the Fisher information matrix; Lynch and Walsh, 1998) and confidence intervals for functions of these parameters must be obtained with approximate methods (eg Podolsky and Holtsford, 1995; Waldmann and Andersson, 1998). Another important advantage with Bayesian methods is that many complex 
hierarchical model structures that earlier were intractable can now be easily investigated (Robert, 2001; Gelman et al, 2004). A Bayesian method for estimation of population genetic structure and $F_{\mathrm{ST}}$ on the basis of multilocus molecular markers was recently presented (Corander et al, 2003, 2004). This method considers the number of populations as an unknown quantity and determines the posterior probabilities of the structure configurations. Moreover, rather than conditioning the $F_{\mathrm{ST}}$ estimate on a single structure it provides modelaveraged (robust) estimate for $F_{\mathrm{ST}}$, where individual $F_{\mathrm{ST}}$ estimates from different population structures are weighted according to corresponding posterior probabilities (of the structure). For other approaches, into Bayesian $F_{\mathrm{ST}}$ estimation, see Holsinger (1999) and Balding (2003).

In this study, we develop a Bayesian method for estimation of the differentiation of the additive genetic variance between populations. The method is applicable to single quantitative traits that can be assumed to have an additive genetic basis. The method uses a Gibbs sampling approach (Gelfand et al, 1995) for estimation of posterior distributions, and CIs are therefore easily obtained for any function of the variance parameters. We first estimate the model averaged neutral $F_{\mathrm{ST}}$ estimate (Corander et al, 2003, 2004) in Pinus sylvestris, and then calculate the weighted $Q_{\mathrm{ST}}$ estimate (according to the posterior probabilities of the hidden structures) and compare these two estimates. Based on the results, we also discuss situations where comparison of the $F_{\mathrm{ST}}$ and $Q_{\mathrm{ST}}$ parameters might be problematic.

\section{Materials and methods}

\section{Estimation and comparison of $F_{\mathrm{ST}}$ and $Q_{\mathrm{ST}}$}

The recently developed Bayesian method for estimation of molecular marker population genetic structure (Corander et al, 2003, 2004) treats both the number of populations and the allele frequencies of the molecular markers of each population as random variables. The posterior distribution of the population structure is estimated from the expression where all allele frequencies have been analytically integrated out. If needed, the posterior sample for the allele frequencies can then be generated afterwards. The accompanying program (BAPS) performs an exact Bayesian analysis by enumerative calculation when the number of original populations is small (less than nine). A Markov Chain Monte Carlo (MCMC) algorithm is used when there are nine or more original populations. Based on the posterior distribution of the structure parameters, a measure of uncertainty regarding the specified populations is obtained for all pairwise comparisons. BAPS can also generate the MCMC samples for the allele frequencies and the $F_{\mathrm{ST}}$ statistic, estimated under all possible population structures that are considered to be likely in the light of the data (model averaged estimate). The empirical samples in this study come from five different locations (original populations) and the enumerative calculations were therefore used for the structure parameter. The length of the MCMC chain for $F_{\mathrm{ST}}$ statistic estimation in BAPS program was based on 50000 iterations (after a burn-in of 10000 had been discarded). For details of this method and the program BAPS, see Corander et al $(2003,2004)$.
Given a polygenic trait with an additive genetic basis and a neutral island model where populations derive from a common ancestor population, it has been shown that the components of between-population variance $\left(\sigma_{b}^{2}\right)$ and the within-population variance $\left(\sigma_{w}^{2}\right)$ can be used to formulate a quantitative trait analog to the molecular $F_{\mathrm{ST}}$ statistic as $Q_{\mathrm{ST}}$ (Prout and Barker, 1989; Spitze, 1993). The molecular $F_{\mathrm{ST}}$ estimate can be used as a neutral drift expectation to which it is possible to compare the $Q_{\mathrm{ST}}$ estimate of a quantitative trait.

Estimation of the $F_{\mathrm{ST}}$ and $Q_{\mathrm{ST}}$ statistics have so far been conducted within the frequentist framework, mainly by using standard methods as ANOVA and REML (an exception is presented in Palo et al, 2003). Rigorous tests for the hypothesis that $F_{\mathrm{ST}}$ and $Q_{\mathrm{ST}}$ are equal are difficult to formulate because these statistics are often estimated from separate analyses conditionally on the original sampling design.

The only relevant way up to now has been to compare an overlap of confidence intervals around the estimates. Confidence intervals can readily be obtained from the ANOVA for balanced designs (Lynch and Walsh, 1998). However, designs are seldom balanced and only approximate confidence intervals can therefore be constructed (eg with the Delta method; Podolsky and Holtsford, 1995; Waldmann and Andersson, 1998). Bootstrap methods can also be used, but the bootstrap can be difficult to implement in multilevel hierarchical designs.

A Bayesian Gibbs sampling approach can be formulated using the hierarchical centering parameterization of Gelfand et al (1995). Consider the following nested random effects linear model

$$
y_{i j k}=\mu+p_{i}+f_{i j}+e_{i j k}
$$

where $y_{i j k}$ is the observed quantitative trait measurement of individual $k$ belonging to family $j$ at population $i, \mu$ the overall mean, $p_{i}$ the population effect at population $i, f_{i j}$ the family effect of family $j$ in population $i$, and $e_{i j k}$ the residual. These parameters are distributed as

$$
p_{i} \sim N\left(0, \sigma_{p}^{2}\right), f_{i j} \sim N\left(0, \sigma_{f}^{2}\right), e_{i j k} \sim N\left(0, \sigma_{e}^{2}\right) .
$$

The hierarchical centering parameterization is obtained by replacing $p_{i}$ with $\gamma_{i}$ and $f_{i j}$ with $\delta_{i j}$, so that $\gamma_{i}=\mu+p_{i}$ and $\delta_{i j}=\mu+p_{i}+f_{i j}$. Hence, $\gamma_{i}$ is centered around $\mu$ and $\delta_{i j}$ is centered around $\gamma_{i}$. This centering has been found to provide a good mixing and convergence properties of the MCMC algorithm (Gelfand et al, 1995). In order to estimate $Q_{\mathrm{ST}}, \sigma_{b}^{2}$ is obtained directly from the population variance $\sigma_{p}^{2}$, that is $\left(\sigma_{b}^{2}=\sigma_{p}^{2}\right)$, whereas the family variance $\sigma_{f}^{2}$ has to be converted into $\sigma_{w}^{2}$ by multiplication of a coefficient $(c)$ that depends on the relationship of individuals within families $\left(\sigma_{w}^{2}=c \sigma_{f}^{2}\right)$. For half-sibs, full-sibs and cloned individuals $c$ is 4,2 and 1 (under the assumption of no dominance and epistasis), respectively. The quantitative trait analog to the molecular $F_{\mathrm{ST}}$ statistic is then estimated as (Prout and Barker, 1989; Spitze, 1993):

$$
Q_{S T}=\frac{\sigma_{b}^{2}}{\sigma_{b}^{2}+2 \sigma_{w}^{2}} .
$$

We implemented this model using WinBUGS14 (Spiegelhalter et al, 2003) and the code is available from the authors. Prior for $\mu$ was taken to be a very flat normal distribution with zero mean and variance $1 / 10^{-6}$. 
Recently, it has been argued that the commonly used inverse Gamma prior is not always uninformative for variance parameters (Gelman et al, 2004). Hence, we performed two separate analyses with Gamma (0.001, $0.001)$ and uniform $\left(10^{+6}, 10^{-6}\right)$ distributions as priors for the (inverse) of the variances $\left(1 / \sigma_{p}^{2}, 1 / \sigma_{f}^{2}\right.$ and $\left.1 / \sigma_{e}^{2}\right)$.

\section{Quantitative and molecular data from $P$. sylvestris}

Seedlings of $P$. sylvestris were grown in a common garden experiment as described by García-Gil et al (2003). The experiment consisted of five populations along a latitudinal cline $\left(67-40^{\circ} \mathrm{N}\right)$. The timing of terminal budset was scored twice per week. A terminal bud was defined as the stage when the stipules of the foliage leaves cover the shoot apex and the youngest foliage leaf are offset from the central axis of the shoot apex. The date of budset was defined as the number of days from sowing to the formation of the bud. The number of families per population was: 22 (Valsaín), 20 (Kolari, Lapinjärvi and Lithuania) and 10 (Puebla de Lillo). In all, 20 individuals per family were scored for the quantitative traits. We assume that the individuals within each family are related as half-sibs (Muona and Harju, 1989; Yang et al, 1996). For the microsatellite work, two individuals per family per population from the common garden experiment were analysed. Ten nuclear microsatellite primers, developed for Pinus taeda, were used to genotype the individuals: PtTX3025, PtTX3013, PtTX2146, PtTX2123 primers (Elsik et al, 2000) and 8846, 4516, 4527, 4528 primers supplied by Dr Auli Karhu. PtTX2146 primer amplified three different polymorphic microsatellite loci named as PtX2146A, PtX2146B and PtX2146C. Out of the ten primers, six microsatellite loci were polymorphic and were used to genotype the 180 individuals. DNA was extracted from needles using Quiagen DNAeasy plant kit. The PCR volume was $25 \mu \mathrm{l}$ and consisted on $50 \mathrm{ng}$ of genomic DNA template, $0.2 \mu \mathrm{M}$ of each primer, $0.2 \mathrm{mM}$ of each dNTP, $2.5 \mu \mathrm{l}$ of $10 \times$ Taq buffer $(500 \mathrm{mM} \mathrm{KCl}$, $100 \mathrm{mM}$ Tris- $\mathrm{HCl}, 1 \%$ Triton X-100, Promega), $2 \mathrm{mM}$ of $\mathrm{MgCl}_{2}$ (Promega) and 2 units of Taq polymerase (Promega). Amplifications were performed using Robocycler gradient 96 (Stratagene). The amplification protocol was: $5 \mathrm{~min}$ at $94^{\circ} \mathrm{C}$; followed by 35 cycles of $1 \mathrm{~min}$ at $94^{\circ} \mathrm{C}, 30 \mathrm{~s}$ at $50^{\circ} \mathrm{C}, 1 \mathrm{~min}$ at $72^{\circ} \mathrm{C}$; and finally one cycle $10 \mathrm{~min}$ at $72^{\circ} \mathrm{C}$. PCR amplifications were resolved using an ABI 377 DNA sequencer and allele scoring was performed by using the GeneScan 3.1 and Genotyper 2.5 softwares.

\section{Results}

\section{P. sylvestris data}

The allele frequencies for the original populations are summarized in Table 1. Analysis with BAPS resulted in two clusters, \{Kolari, Lapinjärvi and Lithuania\} and \{Valsaín and Puebla de Lillo\}, with a posterior probability of 0.994 . The pairwise probabilities, that two populations are equal, are presented in Table 2. The mean of the posterior of the $F_{\mathrm{ST}}$ estimates between the two clusters varied between 0.00650 and 0.0727 for the loci, the overall mean being 0.0316 (95\% CI: 0.0193-0.0438). We also estimated the inbreeding coefficient $\left(F_{\mathrm{IS}}\right)$ within each cluster with the Hickory 1.0 program (Holsinger and Lewis, 2003). The 95\% CI of
Table 1 Microsatellite allele frequencies for the five original Pinus sylvestris populations

\begin{tabular}{|c|c|c|c|c|c|c|}
\hline \multirow[t]{2}{*}{ Locus } & \multirow[t]{2}{*}{ Allele } & \multicolumn{5}{|c|}{ Population } \\
\hline & & Kolari & Lapinjärvi & Lithuania & Valsaín & Puebla de Lillo \\
\hline \multirow[t]{8}{*}{ PtTX3025 } & 214 & 0.03 & 0.03 & 0.03 & 0.00 & 0.17 \\
\hline & 273 & 0.38 & 0.43 & 0.66 & 0.86 & 0.67 \\
\hline & 274 & 0.00 & 0.03 & 0.01 & 0.00 & 0.00 \\
\hline & 279 & 0.17 & 0.03 & 0.06 & 0.07 & 0.03 \\
\hline & 284 & 0.36 & 0.43 & 0.19 & 0.07 & 0.13 \\
\hline & 287 & 0.02 & 0.00 & 0.00 & 0.00 & 0.00 \\
\hline & 294 & 0.00 & 0.01 & 0.00 & 0.00 & 0.00 \\
\hline & 305 & 0.03 & 0.04 & 0.06 & 0.00 & 0.00 \\
\hline \multirow[t]{2}{*}{ PtTX2123 } & 194 & 0.07 & 0.20 & 0.09 & 0.27 & 0.47 \\
\hline & 197 & 0.93 & 0.80 & 0.91 & 0.73 & 0.53 \\
\hline \multirow{2}{*}{ PtTX2146A } & 101 & 0.07 & 0.03 & 0.03 & 0.00 & 0.00 \\
\hline & 103 & 0.93 & 0.97 & 0.97 & 1.00 & 1.00 \\
\hline \multirow{2}{*}{ PtTX2146B } & 126 & 0.22 & 0.11 & 0.07 & 0.11 & 0.06 \\
\hline & 129 & 0.78 & 0.89 & 0.93 & 0.89 & 0.94 \\
\hline \multirow[t]{16}{*}{ PtTX2146C } & 160 & 0.02 & 0.00 & 0.00 & 0.00 & 0.00 \\
\hline & 166 & 0.00 & 0.00 & 0.00 & 0.03 & 0.00 \\
\hline & 177 & 0.02 & 0.02 & 0.00 & 0.17 & 0.06 \\
\hline & 179 & 0.19 & 0.15 & 0.22 & 0.24 & 0.15 \\
\hline & 173 & 0.00 & 0.03 & 0.01 & 0.03 & 0.12 \\
\hline & 186 & 0.02 & 0.00 & 0.00 & 0.01 & 0.00 \\
\hline & 191 & 0.23 & 0.18 & 0.27 & 0.14 & 0.06 \\
\hline & 200 & 0.06 & 0.08 & 0.03 & 0.01 & 0.09 \\
\hline & 206 & 0.00 & 0.00 & 0.00 & 0.01 & 0.00 \\
\hline & 209 & 0.00 & 0.02 & 0.01 & 0.00 & 0.03 \\
\hline & 214 & 0.00 & 0.00 & 0.00 & 0.05 & 0.00 \\
\hline & 217 & 0.42 & 0.48 & 0.35 & 0.29 & 0.47 \\
\hline & 223 & 0.02 & 0.05 & 0.08 & 0.00 & 0.00 \\
\hline & 229 & 0.00 & 0.00 & 0.01 & 0.00 & 0.00 \\
\hline & 232 & 0.00 & 0.00 & 0.00 & 0.00 & 0.03 \\
\hline & 244 & 0.03 & 0.00 & 0.01 & 0.01 & 0.00 \\
\hline \multirow[t]{13}{*}{ PtTX8446 } & 152 & 0.27 & 0.18 & 0.16 & 0.14 & 0.04 \\
\hline & 154 & 0.00 & 0.00 & 0.00 & 0.05 & 0.00 \\
\hline & 156 & 0.00 & 0.00 & 0.01 & 0.08 & 0.00 \\
\hline & 162 & 0.05 & 0.15 & 0.07 & 0.02 & 0.04 \\
\hline & 163 & 0.18 & 0.28 & 0.30 & 0.20 & 0.27 \\
\hline & 165 & 0.11 & 0.18 & 0.20 & 0.18 & 0.15 \\
\hline & 167 & 0.16 & 0.09 & 0.16 & 0.06 & 0.08 \\
\hline & 169 & 0.05 & 0.03 & 0.03 & 0.06 & 0.19 \\
\hline & 171 & 0.03 & 0.03 & 0.03 & 0.08 & 0.04 \\
\hline & 173 & 0.06 & 0.01 & 0.03 & 0.05 & 0.12 \\
\hline & 175 & 0.06 & 0.03 & 0.01 & 0.02 & 0.00 \\
\hline & 177 & 0.02 & 0.03 & 0.00 & 0.02 & 0.08 \\
\hline & 179 & 0.00 & 0.00 & 0.00 & 0.08 & 0.00 \\
\hline
\end{tabular}

Table 2 Pairwise posterior probabilities for allele frequencies of two populations being the same

Kolari Lapinjäroi Lithuania Valsaín Puebla
de Lillo

\begin{tabular}{lccll}
\hline Kolari & & & & \\
Lapinjärvi & 1 & & & \\
Lithuania & 1 & 1 & & \\
Valsaín & $6.94 \mathrm{e}-009$ & $6.94 \mathrm{e}-009$ & $6.94 \mathrm{e}-009$ & \\
Puebla de Lillo & 0.00584 & 0.00584 & 0.00584 & 0.994 \\
\hline
\end{tabular}

the posteriors of $F_{\text {IS }}$ overlapped considerably (northern cluster: 0.0405-0.151 and southern cluster: 0.0346-0.200). Consequently, the estimated level of inbreeding was low 


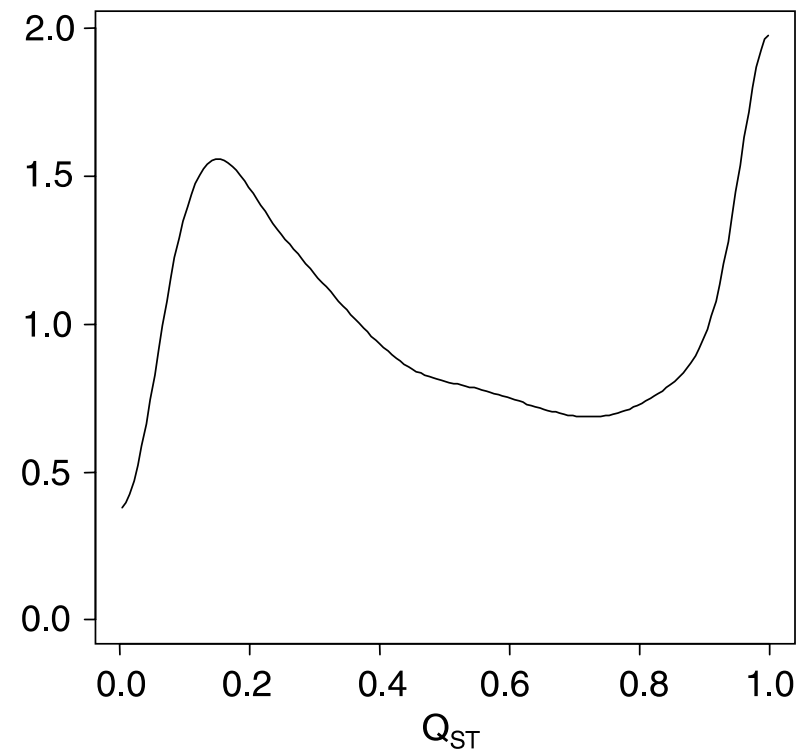

Figure 1 The estimated posterior $Q_{\mathrm{ST}}$ density of date of budset in Pinus sylvestris. The density is based on two parallel Gibbs chains with a burn-in of 50000 and sampling of every 10th iteration yielding a total sample size of 100000 .

(corresponding well with the assumed half-sib relations within families) and did not vary between the two clusters.

Given that the two-cluster configuration in the BAPS analysis had a very high posterior probability, the estimation of the $Q_{\mathrm{ST}}$ differentiation of bud set date was carried out conditionally on this structure. Two parallel MCMC chains were run for 550000 iterations for each type of priors (Gamma, uniform) for variance parameters. The first 50000 iterations were discarded from each chain as burn-in and the chains were thinned by storing every tenth iteration based on autocorrelation plots (plots not shown). The Gelman-Rubin convergence statistic in WinBUGS1.4 strongly supported the conclusion that the chains had converged ( $R$ close to 1$)$ for all variance parameters with both the Gamma and the uniform priors. The two priors seemed to produce identical results, and we will therefore only present the runs based on the uniform priors. The MCMC chains resulted in a posterior $Q_{\mathrm{ST}}$ density that was bimodal with peaks close to 0.2 and 1 (Figure 1). In order to investigate this further, we estimated the family variance $\sigma_{f}^{2}$ for each cluster (extracted from the full model). The northern cluster had a posterior mean of $\sigma_{f}^{2}$ of 125.9 (95\% CI: 110.7145.2), and the southern cluster a posterior mean of $\sigma_{f}^{2}$ of 62.93 (95\%CI: 38.14-97.88). Hence, the within-cluster variation was very different for those two groups. The $\operatorname{Pr}\left(\sigma_{\text {fNorth }}^{2}>\sigma_{\text {fSouth }}^{2}\right)$ was estimated by dividing the number of iterations where $\left(\sigma_{f \text { North }}^{2}>\sigma_{f S o u t h}^{2}\right)$ by the total number of iterations and found to be 0.999 . We also estimated $Q_{\text {ST }}$ based on the original population configuration for comparative purposes. The mean, mode and median were high $(0.765,0.817$ and 0.779 , respectively), but the CI of the posterior was wide (95\% CI: $0.490-0.964)$.

\section{Discussion}

It is shown here how the $Q_{\mathrm{ST}}$ statistic, which is commonly used for estimation of differentiation in quantitative traits, can be formulated in a Bayesian framework. A Gibbs sampling approach with hierarchical centering was used in the estimation, because it has been shown to work well (Gelfand et al, 1995). When the molecular markers from $P$. sylvestris were subjected to the BAPS program, it was found that the estimated neutral population genetic structure consists of two clusters that correspond very well to the north/south geographic distribution. However, when trying to estimate $Q_{\mathrm{ST}}$ of the bud set data from $P$. sylvestris, the MCMC-chains produced a bimodal posterior of $Q_{\text {ST }}$. The evident reason for the bimodality is that the family variances differed considerably between the two clusters because of the strong cline within the northern cluster (García-Gil et al, 2003). An earlier study has found that microsatellites and other molecular markers are hardly differentiated at all, whereas timing of bud set is very different between the original populations of the northern cluster (Karhu et al, 1996). Consequently, the assumption of variance homogeneity at the family level is violated and no comparison between the $Q_{\mathrm{ST}}$ and the $F_{\mathrm{ST}}$ estimates can therefore be made.

\section{Statistical and evolutionary assumptions behind $Q_{\mathrm{ST}}$}

One of the critical assumptions when estimating $Q_{\mathrm{ST}}$ is that the populations are evolving at the same rate, that is, that genetic drift is converting the family variance within populations to the between-population component at the same rate. It is therefore of fundamental importance to investigate $a$ prior $i$ whether the additive genetic variance (or the heritability) differs considerably between populations. Several of the studies included in recent review articles (Merilä and Crnokrak, 2001; McKay and Latta, 2002) have reported average heritabilities. Since relatively few authors have reported both $Q_{\mathrm{ST}}$ and population-specific heritability estimates in their studies, it is difficult to evaluate if heterogeneity in within-population variance is a common problem.

Another assumption that is more challenging to verify is that the molecular markers and the genes of the quantitative traits should have the same mutation rates. Although it is inherently difficult to estimate proper mutation rates of both molecular markers and quantitative traits, it has been suggested that different marker types can have considerably dissimilar mutation rates (Balloux et al, 2000). Moreover, theoretical studies have shown that the $F_{\mathrm{ST}}$ statistics is very sensitive to variations in the mutation rate ( $\mathrm{Fu}$ et al, 2003) and to unequal migration rates between populations (Wilkinson-Herbots and Ettridge, 2004).

The level of inbreeding was low and did not differ between the northern and southern clusters. Thus, the bias should be small for the assumption of no inbreeding when estimating $Q_{\mathrm{ST}}$. Theoretically, it is possible to derive a Bayesian $Q_{\mathrm{ST}}$ statistic that takes inbreeding into account. Prior information from the level of inbreeding could easily be attained from molecular markers. Unfortunately, it is practically much more difficult to specify a model for the dominance variance components that are introduced by inbreeding (De Boer and Hoeschele, 1993). Hence, we did not try to implement an inbreeding $Q_{\mathrm{ST}}$ for this data set.

In Waldmann and Andersson (1998), heritability estimates varied between populations considerably for 
some traits (flowering date in Scabiosa canescens varied between 0.098 and 1.49), but not that much for others (flowering date in S. columbaria varied between 0.198 and 0.450). However, the confidence intervals of the heritabilities were wide in that study and overlaps between populations were common. Large variation in population-specific heritability levels were also found for some traits in two rare plants, whereas some traits displayed very similar heritability levels between populations (Petit et al, 2001). A similar result was found by Widen et al (2002) for Brassica cretica. The within-population genetic variance varied between 0.111 and 11.0 for internode length, whereas node number only varied between 12.9 and 64.4 between populations of this species.

Moreover, it should also be noticed that the (frequentist) ANOVA and REML methods produce point estimates for the variance components even in the presence of considerable variance heterogeneity. For example, an REML analysis of the bud set data in this study produced a $Q_{\text {ST }}$ estimate of 0.274 (when estimated conditional on the northern and southern cluster structure). An obvious indication for variance heterogeneity can be obtained by checking that the residuals do not follow a straight line in the Normal Quantile-Quantile plot (Figure 2; see also Pinheiro and Bates, 2000).

Recently, a theoretical study by Lopez-Fanjul et al (2003) showed that $Q_{\mathrm{ST}}$ can be severely biased if there are nonadditive gene actions (dominant and/or epistatic loci contribute to the phenotype). Hence, comparison of $Q_{\mathrm{ST}}$ and $F_{\mathrm{ST}}$ for inference of the relative importance of drift and selection in population differentiation is limited to purely additive traits. In this study, we have assumed that individuals within families are related as half-sibs, which seems to be reasonable when considering the mating system of $P$. sylvestris (Muona and Harju, 1989;

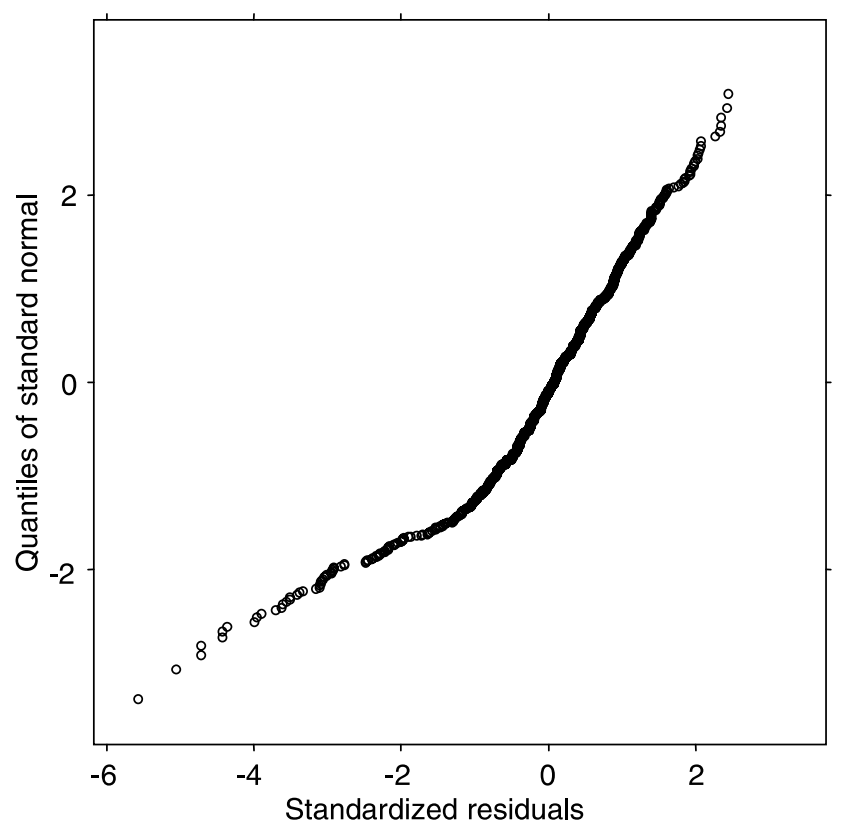

Figure 2 Normal Quantile-Quantile plot of standardized residuals from an REML analysis of the budset data using the northern and southern clusters as population levels. Normally distributed residuals should display a straight line. This plot clearly shows two different linear trends.
Yang et al, 1996) and the $F_{\mathrm{IS}}$ result attained with Hickory. However, it is possible that a small fraction of full-sibs are present and introduce a small amount of error due to dominance (Lynch and Walsh, 1998).

So far, no theoretical investigation has been undertaken on how different selection regimes within populations influence $Q_{\mathrm{ST}}$. Although it has been shown that migration (or pollen dispersal) between local populations in which selection favours different trait values can maintain substantial amounts of genetic variation at the between-population level (Barton and Keightley, 2002). Regarding our data, one could suspect that divergent selection is more prevalent within the northern cluster, and that uniform selection (or drift) is the dominating force within the southern cluster where original populations occur in a rather similar environment (latitude). In fact, analyses of bud set date at the within-cluster level (using initial populations) with WinBUGS14 revealed that mean $Q_{\mathrm{ST}}$ was 0.728 for the northern cluster and 0.0979 for the southern cluster (these estimates should only be taken as very approximate because CIs were very wide).

\section{Differentiation in pine and other forest trees}

Two recent studies have compared differentiation in molecular markers and quantitative traits in different Pinus species. Yang et al (1996) compared allozyme differentiation and quantitative genetic differentiation in $P$. contorta ssp. latifolia and found that specific gravity, stem diameter, stem height and branch length had significantly higher $Q_{\mathrm{ST}}$ (between 0.133 and 0.195 ) than $F_{\mathrm{ST}}(0.019)$ values. In $P$. pinaster (Gonzalez-Martínez et al, $2002)$, the $Q_{\mathrm{ST}}$ values were very high for stem form, total height growth and survival at 30 years age $(0.973,0.791$ and 0.732 , respectively), and significantly higher than the allozyme $F_{\mathrm{ST}}(0.048)$. However, Gonzalez-Martinez et al (2002) also report considerably lower $Q_{\mathrm{ST}}$ estimates $(0.12$ and 0.20 ) for height growth of two other pine species from the Mediterranean.

Strong diversifying selection is apparent in several widespread tree species as $Q_{\mathrm{ST}}$ values often are much higher than neutral $F_{\mathrm{ST}}$ values, especially in timing of bud burst (Le Corre and Kremer, 2003). However, the $Q_{\mathrm{ST}}$ values are not always extremely high. Instead, it is the $F_{\mathrm{ST}}$ values that are very low. This is not surprising because many tree species are often wind pollinated and distributed over large areas. For example, a recent study in Picea glauca revealed that $Q_{\mathrm{ST}}$ estimates of 10 traits ranged between 0.035 and 0.246 (Jaramillo-Correa et al, 2001). Of those were only 8 year height $\left(Q_{\mathrm{ST}}=0.082\right)$, 13 year height $\left(Q_{\mathrm{ST}}=0.069\right)$, total wood density $\left(Q_{\mathrm{ST}}=0.102\right)$ and date of budset $\left(Q_{\mathrm{ST}}=0.246\right)$ higher than the neutral differentiation of allozymes $\left(G_{S T}=0.014\right)$ and ESTPs $\left(G_{S T}=0.014\right)$. Unfortunately, no heritability estimates were reported for the separate populations.

In two other theoretical studies (Latta, 1998; Le Corre and Kremer, 2003), the differentiation at neutral molecular markers, the QTL behind the trait and the adaptive trait itself was compared under different selection regimes and with different levels of gene flow. Their general conclusion was that it is more common that population differentiation shows pattern of genetic variability that differs between markers, QTL and the adaptive trait, than that the differentiation is the same. 
Le Corre and Kremer (2003) found that the highest disparity between the three levels occurred under highly diversifying selection and high gene flow, a situation that corresponds very well with the pine biology.

In conclusion, we have presented a Bayesian method for estimation of $Q_{\mathrm{ST}}$ that can utilize the information regarding the actual population structure estimated using neutral molecular markers. The method should work well when the quantitative traits of the populations differentiate at the same rate (ie have similar variances). However, when the heritability differs substantially between populations, the MCMC estimation may result in bimodal posteriors. This problem was illustrated by applying the methods to a data set of date to bud set in $P$. sylvestris. We also recommend that future studies in addition to presenting $Q_{\mathrm{ST}}$ estimates, also present between-family variance or heritability estimates for each trait and population. Moreover, when using ANOVA and REML methods, it is of particular importance to check that the residuals are normally distributed and do not follow any deviating trends. Finally, many evolutionary forces can potentially bias $F_{\mathrm{ST}}$ and $Q_{\mathrm{ST}}$ comparisons and they should therefore be interpreted with care.

\section{Acknowledgements}

This work was supported by Academy of Finland (Grant no. 202324) and the Marie Curie fellowship (QLK5-CT2000-51233) under the 5th Framework Programme. We are grateful to Dr O Savolainen who provided us a $P$. sylvestris data set, which have been collected under the support of the Environmental and Natural Resources Research Council of Finland (ES1587).

\section{References}

Avise JC (1993). Molecular Markers, Natural History and Evolution. Kluwer Academic Publishers: Boston, MA.

Balding DJ (2003). Likelihood-based inference for genetic correlation coefficients. Theor Popul Biol 63: 221-230.

Balloux F, Brünner H, Lugon-Moulin N, Hausser J, Goudet J (2000). Microsatellites can be misleading: an empirical and simulation study. Evolution 54: 1414-1422.

Barton NH, Keightley PD (2002). Understanding quantitative genetic variation. Nat Rev Genet 3: 11-21.

Beaumont MA, Rannala B (2004). The Bayesian revolution in genetics. Nat Rev Genet 5: 251-261.

Corander J, Waldmann P, Marttinen P, Sillanpää MJ (2004). BAPS 2: enhanced possibilities for the analysis of genetic population structure. Bioinformatics 20: 2363-2369.

Corander J, Waldmann P, Sillanpää MJ (2003). Bayesian analysis of genetic differentiation between populations. Genetics 163: 367-374.

De Boer IJM, Hoeschele I (1993). Genetic evaluation methods for populations with dominance and inbreeding. Theor Appl Genet 86: 245-258.

Elsik CG, Minihan VT, Hall SE, Scarpa AM, Williams CG (2000) Low-copy microsatellite markers for Pinus taeda L. Genome 43: 550-555.

Excoffier L (2001). Analysis of population subdivision. In: Balding DJ, Bishop M, Cannings C (eds) Handbook of Statistical Genetics. Wiley: NY. pp 271-307.

$\mathrm{Fu} \mathrm{R}$, Gelfand AE, Holsinger KE (2003). Exact moment calculations for genetic models with migration, mutation, and drift. Theor Popul Biol 63: 231-243.
García-Gil MR, Mikkonen M, Savolainen O (2003). Nucleotide diversity at two phytochrome loci along a latitudinal cline in Pinus sylvestris. Mol Ecol 12: 1195-1206.

Gelfand AE, Sahu SK, Carlin BP (1995). Efficient parametrizations for normal linear mixed models. Biometrika 82: 479-488.

Gelman A, Carlin JB, Stern HS, Rubin DB (2004). Bayesian Data Analysis 2nd edn. Chapman \& Hall: London.

Goldstein DB, Schlötterer C (1999). Microsatellites: Evolution and Applications. Oxford University Press: Oxford, UK.

Gonzalez-Martínez SC, Alia R, Gil L (2002). Population genetic structure in a Mediterranean pine (Pinus pinaster Ait.): a comparison of allozyme markers and quantitative traits. Heredity 89: 199-206.

Holsinger KE (1999). Analysis of genetic diversity in geographically structured populations: a Bayesian perspective. Hereditas 130: 245-255.

Holsinger KE, Lewis PO (2003) Hickory. Ver. 1.0. Department of Ecology and Evolutionary Biology, University of Connecticut, CT. Available via http://www.eeb.uconn.edu/.

Jaramillo-Correa JP, Beaulieu J, Bousquet J (2001). Contrasting evolutionary forces driving populations structure at expressed sequence tag polymorphisms, allozymes and quantitative traits in white spruce. Mol Ecol 10: 2729-2740.

Karhu A, Hurme P, Karjalainen M, Karvonen P, Karkkainen K, Neale D et al (1996). Do molecular markers reflect patterns of differentiation in adaptive traits of conifers? Theor Appl Genet 93: 215-221.

Lande R (1992). Neutral theory of quantitative genetic variance in an island model with local extinction and colonization. Evolution 46: 381-389.

Latta RG (1998). Differentiation of allelic frequencies at quantitative trait loci affecting locally adaptive traits. Am Nat 151: 283-292.

Le Corre V, Kremer A (2003). Genetic variability at neutral markers, quantitative trait loci and trait in a subdivided population under selection. Genetics 164: 1205-1219.

Lopez-Fanjul C, Fernandez A, Toro MA (2003). The effect of neutral nonadditive gene action on the quantitative index of population divergence. Genetics 164: 1627-1633.

Lynch M (1994). The neutral theory of phenotypic evolution. In: Real L (ed) Ecological Genetics. Princeton University Press: Princeton, NJ. pp 86-108.

Lynch M, Walsh B (1998). Genetics and Analysis of Quantitative Traits. Sinauer Associates: Sunderland, MA.

McKay JK, Latta RC (2002). Adaptive population divergence: markers, QTL, and traits. Trends Ecol Evol 17: 285-291.

Merilä J, Crnokrak P (2001). Comparison of genetic differentiation at marker loci and quantitative traits. J Evol Biol 14: 892-903.

Muona O, Harju A (1989). Effective population sizes, genetic variability, and mating system in natural stands and seed orchards of Pinus sylvestris. Silvae Genetica 38: 221-228.

Palo JU, O'Hara RB, Laugen AT, Laurila A, Primmer CR, Merilä $\mathrm{J}$ (2003). Latitudinal divergence of common frog (Rana temporaria) life history traits by natural selection: evidence from a comparison of molecular and quantitative genetic data. Mol Ecol 12: 1963-1978.

Petit C, Freville H, Mignot A, Colas B, Riba M, Imbert E et al. (2001). Gene flow and local adaptation in two endemic plant species. Biol Cons 100: 21-34.

Pinheiro J, Bates DM (2000). Mixed Effects Models in S and S-Plus. Springer-Verlag: New York.

Podolsky RH, Holtsford TP (1995). Population structure of morphological traits in Clarkia dudleyana. I. Comparison of FST between allozymes and morphological traits. Genetics 140: 733-744.

Prout T, Barker JSF (1989). Ecological aspects of the heritability of body size in Drosophila buzzatii. Genetics 123: 803-813.

Reed DH, Frankham R (2001). How closely related are molecular and quantitative measures of genetic variation? A meta-analysis. Evolution 55: 1095-1103. 
Robert CP (2001). The Bayesian Choice. Springer-Verlag: New York 2nd edn.

Spiegelhalter DJ, Thomas A, Best N, Lunn D (2003) WinBUGS. Ver. 1.4 user manual. MRC Biostatistics Unit, Cambridge, U.K. Available via http://www.mrc-bsu. cam.ac.uk/bugs.

Spitze K (1993). Population structure in Daphnia obtusa: quantitative genetic and allozyme variation. Genetics 135: 367-374.

Waldmann P, Andersson S (1998). Comparison of quantitative genetic variation and allozyme diversity within and between populations of Scabiosa canescens and S. columbria. Heredity 81: 79-86.

Weir BS, Hill WG (2002). Estimating F-statistics. Ann Rev Genet 36: 721-750.
Whitlock MC (1999). Neutral additive genetic variance in a meta-population. Genet Res 74: 215-221.

Widen B, Andersson S, Rao G-Y, Widen M (2002). Population divergence of genetic (co)variance matrices in a subdivided plant species, Brassica cretica. J Evol Biol 15: 961-970.

Wilkinson-Herbots HM, Ettridge R (2004). The effect of unequal migration rates on $\mathrm{F}_{\mathrm{ST}}$. Theor Popul Biol 66: 185-197.

Wright S (1951). The genetical structure of populations. Ann Eugen 15: 323-354.

Wright S (1965). The interpretation of population structure by $F$-statistics with special regard to system mating. Evolution 19: 395-420.

Yang RC, Yeh FC, Yanchuck AD (1996). A comparison of isozyme and quantitative genetic variation in Pinus contorta ssp. latifolia by Fst. Genetics 142: 1045-1052. 